\title{
Hermetischer Kosmos: Aby Warburg und Roberto Longhi in Ferrara
}

Ein so großer Geist wie Goethe und alles falsch.

Thomas Bernhard

Wenn die Namen Aby Warburgs und Roberto Longhis im Titel dieses Beitrags figurieren, dann, um zunächst jene Grenzlinie zu markieren, die zwischen der italienischen und der deutschsprachigen Kunstgeschichte verläuft und die sich wohl besser nicht beschreiben läßt, als im Abstand zwischen den in ihrer Zeit und ihrer jeweiligen Wissenskultur vielleicht folgenreichsten Protagonisten ihres Faches. Daß es sich um eine überaus deutliche Demarkation handelt, wird in der Aufrufung des Kulturwissenschaftlers und Ikonologen Warburg auf der einen und der des virtuosen Stil- und Formanalytikers Longhi auf der anderen Seite evident; die methodische Dialogstille zwischen der deutschen und der italienischen kunsthistorischen Denkschule besitzt hier ihre Pole.

Und doch bestehen Analogien in Haltung und Perspektive, die größere Gemeinsamkeiten benennen lassen, als es die gängige Opposition suggeriert. Schon daß sich beide, lange bevor Ferrara zu einem kapitalen Gegenstand der Forschung wurde, der Kunst der estensischen Residenz widmeten, signalisiert Korrespondenzen. Der Begriff ,hermetisch' erscheint hier, um auf den, stellenweise undurchdringlichen, fast okkulten Charakter anzuspielen, der sowohl Warburgs als auch Longhis Schriften zur Ferraresischen Kunst attestiert worden ist. Zugleich wird mit ihm auf die durchaus eigene Ausprägung der Ferrareser Malerei des 15. Jahrhunderts angespielt; eine Malerei, deren expressive Outriertheit und plastische Diesseitigkeit sie immer wieder als irritierende ,Sonderform' der italienischen Renaissance haben begreifen und sie gelegentlich unter den unmittelbaren Einfluß, oder doch wenigstens in die Nachbarschaft der deutschen Renaissance haben stellen lassen. Deshalb auch wird Ferrara hier zugleich ein Kosmos genannt, weil es durchaus eine eigene, kunsthistorische ,Weltordnung، ist, die die Ferraresische Kunst, zumal jene des 15. Jahrhunderts, Zeit ihrer unverwechselbaren Eigenheit, auszeichnet. 
Dieser Kosmos freilich ist vornehmlich ein kunstliterarisches Konstrukt. Und seine Autoren sind Aby Warburg und Roberto Longhi. Festgeschrieben wurden die Gesetze dieser Gemarkung in Warburgs Aufsatz Italienische Kunst und Internationale Astrologie im Palazzo Schifanoja zu Ferrara von $1912^{1}$ und in Longhis Officina Ferrarese von $1934^{2}$ (mit späteren, aggiornierten Ausgaben).

Warburgs Aufsatz muß nicht vorgestellt werden; er ist gleichsam zum Gründungstext einer expansiv sich erneuernden Kunstwissenschaft am Beginn des 20. Jahrhunderts geraten und bis in die jüngste Zeit deren vorzüglicher Referenztext geblieben. ${ }^{3}$ Warburg war ein notorischer Grenzverletzer; sowohl was sein Fach, als auch, was die Kulturen betrifft. Seit etwa 1905 hat er sich intensiv mit der Geschichte der Mythographie und der Astrologie beschäftigt; und zwar über einen rein antiquarischen Impetus hinaus, indem er die Renaissance insgesamt als eine Rückkehr göttlich inspirierter Aufklärung auffaßte, und über dieses Interpretament wiederum einen unmittelbaren Zeitbezug hergestellt hat.

Es ist besonders bemerkenswert, daß Warburgs vielleicht folgenreichster Beitrag dazu im Kontext eines in Italien ausgerichteten internationalen Kunsthistorikertreffens vorgetragen wurde. Gemeinsam mit Adolfo Venturi traf Warburg im Jahr 1912 Vorbereitungen für einen internationalen KunsthistorikerKongreß in Rom, der sich mit der italienischen Kunst und ihrem Verhältnis zu anderen Ländern beschäftigte. Er sollte Warburg - neben dem von ihm wiederholt angestellten Versuch, durch Kongreßteilnahme seiner akademischen Isolation zu begegnen - die Möglichkeit bieten, die von ihm stets eingeklagten Grenzüberschreitungen selbst zu begehen: hier, die Grenzziehung zwischen Italien und Deutschland wirkungsvoll zu durchbrechen und zugleich die Epochen - von der Spätantike über das Mittelalter bis in die Neuzeit - miteinander zu versöhnen.

In seinem Vortrag und dem später veröffentlichten Aufsatz deutete Warburg - gemäß seiner Auffassung der Renaissance als Epoche der Wiederauflebung des heidnischen Götterhimmels in seiner ursprünglichen Menschlichkeit und Schönheit - den ersten Dekan im Sternzeichen des Widders, jenen dunkelhäutigen Mann also, dessen Gewand mit einem Seil gegürtet ist, als ein griechisches Urbild, oder vielmehr als eines, das, entstellt und verzerrt und kaum noch

1 Aby Warburg: Italienische Kunst und internationale Astrologie im Palazzo Schifanoja zu Ferrara (1912), in: ders., Die Erneuerung der heidnischen Antike. Kulturwissenschaftliche Beiträge zur Geschichte der europäischen Renaissance, wieder abgedruckt in: Gesammelte Schriften. Studienausgabe, mit einem Vorwort v. Horst Bredekamp/Michael Diers, Bd. I, 1.2, Berlin 1998, 459-481.

2 Roberto Longhi: Officina Ferrarese (1934), Florenz ${ }^{9} 1975$.

3 Vgl. Andreas Beyer: 78 Jahre danach. Zur Geistesgegenwart der Ikonologie, in: Horst Bredekamp/Michael Diers/Charlotte Schoell-Glass (Hrsg.), Aby Warburg. Akten des internationalen Symposiums Hamburg 1990, Schriften des Warburg-Archivs im Kunstgeschichtlichen Seminar der Universität Hamburg, Bd. 1, Weinheim 1991, 269-279. 
als dieses erkennbar, in ein solches zurückübersetzt werden konnte. Es handele sich, so Warburg, um ein Bild der klassischen Antike, das in die indische Astrologie gefunden und über einen arabischen Gelehrten des neunten Jahrhunderts (Abu Ma'shar) auf den Wänden des Ferrareser Palasts wieder nach Europa zurückgefunden habe.

Warburgs These, wonach es sich bei dieser Gestalt um nichts anderes handele als um eine verfremdete Version des Perseus, der am Nachthimmel in der Nähe des Widder aufgeht, hat schon die eingeweihten Kenner der Materie unter seinen Zeitgenossen (namentlich Fritz Saxl) nicht überzeugen können. Gleichwohl hat das der wissenschaftlichen ,Karriere‘ des Textes kaum geschadet; nach Ernst H. Gombrich nämlich sei der Deutungsversuch über Schifanoia "[... interessant als Extrembeispiel der Warburgschen Methode. ${ }^{\text {" }}$ Gombrichs Analyse von Warburgs astrologischen Deutungen hat Kristen Lippincott wiederum noch jüngst als die treffendste und gültige Einschätzung dieser Arbeit gewürdigt: „[...] the best study [that] remains"; die helfe, „[...] to set Warburg's writings on astrological iconography into a convincing intellectual framework.".

Gombrich argumentiert, daß Warburgs Rang ,in der Kunstgeschichte erst dann in seiner wirklichen Bedeutung gesehen werden“ könne, wenn man „seine hartnäckige Weigerung, sich mit Stilfragen auseinanderzusetzen, ganz deutlich“ mache: Nach allgemeiner Auffassung stünde Warburg in der Kunstgeschichte für die Aufgabe der Formenanalyse zugunsten der Ikonographie: „[...] seine Interpretation des astrologischen Bilderzyklus im Palazzo Schifanoja in Ferrara war zwar in der Tat ein Höhepunkt seines öffentlichen Schaffens, aber ihn interessierte weniger die Identifizierung der Dekane als die Entdeckung umgeformter und verkleideter Gestalten der Antike, die in ihrer unverfälschten Schönheit wiederhergestellt sein wollten." Wenn Warburg sich der Ikonographie zuwandte, so Gombrich, dann nur aus pädagogischen Erwägungen heraus. Er habe der degradierten Form eines Themas gerne seine ursprüngliche Fassung gegenüber gestellt, ,[...] um so das Erlebnis der Befreiung bestimmter Inhalte von fremden Zusätzen innerlich mitzumachen." Für ihn habe die Ikonologie nicht das Studium komplexer Embleme und Allegorien dargestelit, „sondern die Untersuchung der Wechselwirkung von Form und Inhalt im Konflikt der Traditionen."

4 Ernst H. Gombrich: Aby Warburg. Eine intellektuelle Biographie, Frankfurt a. M. 1981, 261.

5 Kristen Lippincott: Urania redux. A View of Aby $W$ arburg's $W$ ritings on Astrology and Art, in: Richard Woodfield (Hrsg.), Art History as Cultural History. Warburg's Projects, Amsterdam 2001, 157.

6 Gombrich 1981 (wie Anm. 4), 419.

7 Gombrich 1981 (wie Anm. 4), 419. 
Hier, so Gombrich, nähere man sich „[...] der Definition dessen, was zu dem Geheimnis von Warburgs pädagogischem Erfolg beigetragen hat. Indem er den stilgeschichtlichen Ansatz ablehnte bzw. ignorierte", habe dieser das Hauptthema der theoretischen Kunstgeschichte umgangen, das letztlich auf Winckelmann und Hegel zurückginge, nämlich das Problem eines einheitlichen Stils als Ausdruck eines ,Zeitalters“. Also die „Auffassung, daß der Zeitgeist sich in parallelen Erscheinungsformen äußere, wobei die Verbindung zwischen Kunst und Weltanschauung am häufigsten erörtert wurde. ${ }^{68}$ In diesem Zusammenhang kommt Gombrich auf Warburgs Opposition gegen solchen Zeitgeist zu sprechen, wie sich das etwa am Beispiel von dessen Betonung der unvergleichlichen Individualität Rembrandts artikuliere. „Es ging ihm nicht um Stile, die sich nach bestimmten Gesetzmäßigkeiten entfalten, sondern um Individuen, die in Konflikte und Entscheidungen verwickelt waren. [...] Durch die Konzentration auf einen bestimmten Auftrag sowie auf die Lösung, die aus den gegensätzlichen Möglichkeiten einer historischen Situation hervorgeht, errang Warburgs Ansatz seinen größten Triumph. Er nötigte den Kunsthistoriker, die Allgemeinheiten der, Geistesgeschichte' aufzugeben und sich auf einzelne Menschen und einzelne Bilder zu konzentrieren." Nun wird aber zu fragen sein, ob Warburgs Ansatz, so wie er sich zumal in seiner Deutung der Schifanoia-Fresken manifestiert, damit auch nur annähernd treffend bezeichnet ist.

Kristen Lippincott hat unlängst nachgewiesen, daß Warburgs Thesen zum Palazzo Schifanoia drei wesentliche Irrtümer aufweisen:

1. Wenngleich es eine ikonographische Linie zwischen einer Figur in den von Warburg herangezogenen Quellen (also der Tabula Bianchini, dem Astrolabium planum) und dem Dekansbild in Schifanoia gäbe, sei doch keine dieser Figuren, streng genommen, ein Dekan-Gott, - jedenfalls nicht im eigenen Recht. Vielmehr stellten diese ,Masken' des Planeten dar. ${ }^{10}$

2. Bei der Identifizierung des Schifanoia-Dekans mit Perseus handele es sich um einen Irrtum, der einer mißverständlichen Lektüre von Franz Bolls Studie über die griechischen Texte der Sphaera barbarica geschuldet sei; eine Arbeit, auf die Warburg in seinem Text rekurriert. Boll hatte ein oder zwei isolierte Phäno-

8 Gombrich 1981 (wie Anm. 4), 419-420.

9 Gombrich 1981 (wie Anm. 4), 420.

10 Lippincott 2001 (wie Anm. 5), 159 ff. Zur weiteren Erforschung der Ferrareser Fresken vgl. Marco Bertozzi: La tirannia degli astri. Aby Warburg e l'astrologia di Palazzo Schifanoja, Bologna 1985; Marco Bertozzi: Schifanoja. Il salone dei dipinti perduti. Con una appendice su Aby Warburg: lo ,stile' del paganesimo antico, in: Elena Monatti (Hrsg.), Lo Zodiaco del Principe. I Decani di Schifanoja di Maurizio Bonora, Ferrara, 1992, 23-33; Marco Bertozzi (Hrsg.): Aby Warburg e le metamorfosi degli dèi antichi, Ferrara 2002. 
Hermetischer Kosmos: Aby Warburg und Roberto Longhi in Ferrara



(ictïlschtes I.onghi-Zitat won D)arid I Iockne!: 2001 
mene in den ägyptischen Tierkreiszeichen ausfindig machen können, die von den gängigeren graeco-römischen Modellen beeinflußt sein könnten. Warburg schloß daher aus diesen zwei sehr marginalen Punkten, daß es eine Verbindung zwischen der griechischen und der ägyptischen Darstellung der Dekane geben müsse, während es Bolls ausgesprochene Absicht war, eben nicht Traditionskontinuität aufzuzeigen, sondern, im Gegenteil, nachzuweisen, wie wenig beide Systeme miteinander zu tun hatten. Da fällt schon weniger ins Gewicht, daß aus Bolls Text deutlich hervorgeht, daß die graeco-römischen und ptolemäischen Konstellationen, die mit dem ersten Dekan des Widders erscheinen, Cepheus und Eridanus sind und nicht Perseus. Jedenfalls gibt es weder eine antike noch eine mittelalterliche Quelle, die Perseus mit dem ersten Dekan des Aries identifiziert. ${ }^{11}$

3. Stützte Warburg sich bei seiner Deutung auf die lateinischen Übersetzungen des arabischen Astrologen Abu Ma'shar und damit auf kontaminierte Kompilationen - namentlich auf jene des Georgius Zotori Zapari Fenduli, der seinerseits auf Hermannus Dalmata zurückgeht ${ }^{12}$ - um die Wanderung der Textvorlage und ihre Verwendung in Ferrara plausibler zu machen. In der Originalfassung sind allerdings sämtliche als iuxta Indos bezeichneten - indischstämmigen - Bilder ohne irgendeinen Bezug zur klassischen Tradition und gänzlich einer lokalen, indischen astro-mythologischen Tradition entsprungen. Warburg hatte demnach zwar Recht damit, in dieser Beschreibung jene des ersten Dekans zu erkennen, nur hat eben diese Figur keinerlei Beziehungen zu irgendeiner Bildtradition der europäischen Antike. ${ }^{13}$

Das ist mißlich, präsentierte Warburg ja gerade diesen Dekan gleichsam als Emblem von Wissenschaft und Aufklärung, was Lippincott zu der Äußerung veranlaßt: „There is a certain sadness in all this. " 14 Man wird aber, statt diesem Umstand Traurigkeit zu attestieren, besser daran tun, „Herrn Meisters“ Ausruf in Thomas Bernhards Komödie Über allen Gipfeln ist Rub (1981) zu zitieren: "Ein so großer Geist wie Goethe und alles falsch.“

,Falsch' freilich nur, wenn man Gombrichs Charakterisierung folgt, der Warburg ja unterstellt hat, den Einzelfall gegen das Allgemeine auszuspielen. Dann bliebe wirklich wenig von dessen Deutung der Schifanoia-Fresken zu retten. Tatsächlich aber geht Warburg, ganz im Gegenteil, und im Falle von Ferrara besonders, von einem ganzheitlichen Gedanken aus, sucht er nicht Abgrenzung und Isolation, sondern vielmehr Fermente der Kontinuität. Seine 
grundlegende Erkenntnis, wonach die Frührenaissance in einem langwierigen, quälenden Prozeß den heiteren Götterhimmel des Altertums erst aus der spätantiken scholastischen und bilderlosen Gelehrsamkeit und erstarrter Heraldik hat entschälen müssen, beharrt auf letztlich ungebrochenen, allenfalls verschütteten Linien: Archetypische Grundstrukturen einer ,Kulturpsychologie', nicht die Abweichungen davon, werden hier verfolgt. Und zumal die Astrologie - die Warburg, und nach ihm Fritz Saxl und Erwin Panofsky, als unausgesetzte Bilderwanderung beschrieben haben, die über viele Stationen, in Babylon ihren Anfang nehmend, sich im und vom Abendland aus verbreitend und über arabische und indische Wege endlich nach Europa zurückkehrend - galt da als Beleg einer ungebrochenen Wirkkraft der antikischen Heilsgewißheit. Diese selbst in der enigmatischen Figur des Dekans des Ferrareser Freskos, in der vermeintlichen Figur des Perseus noch auffinden zu wollen, läßt daher Warburgs berühmtes Diktum, wonach der „liebe Gott im Detail“ stecke, auch so lesen: noch im Detail äußere sich der universelle Bezug, verberge sich Gott!

Warburgs Perspektive hat gerade darin folgenreich gewirkt. In einem gemeinsamen Aufsatz haben Erwin Panofsky und Fritz Saxl 1933 die astronomisch-astrologischen Bildtraditionen im Rahmen der Antikenrezeption abgehandelt. ${ }^{15}$ Seit diesen Arbeiten, so jüngst Dieter Blume, herrsche „ [...] das Mißverständnis, es ginge bei astronomisch-astrologischen Bildern im wesentlichen um Antikenrezeption." Panofsky und Saxl etwa reduzierten den gesamten Problemkreis der astrologischen Bilderwelt auf einen Teilaspekt im Nachleben der Antike - was die weitere Beschäftigung mit diesem für das profane Denken so wichtigen Fragenkreis „eher behindert als befruchtet" habe, weil es sich im Grunde um ein sehr formales System handele, „das die Inhalte der Bilder allein auf ihre angeblich antike Herkunft befragt und ihre Formen auf die größere oder geringere Antikennähe hin untersucht. " Was solchermaßen zu einer Art Schematismus geriet, ist bei Warburg vorbereitet. Und in einer erhellenden, vielleicht auch decouvrierenden Äußerung bekennt Warburg, daß eine „kritische Ikonologie“ „ein fortwährendes Wegräumen unberechenbarer Schichten nicht verständlicher Zutaten" verlange." Warburg insistiert tatsächlich auf Herleitung, Überlieferung und Kontinuität, auf Einschreibung auch des vermeintlich Fremden in ein teleologisches Prinzip, das in der Wiederauflebung der antiken Götterwelt die Befreiung des Geistes und der Schönheit feiert. So gerät das astrologische Programm des Palazzo Schifanoia auch zum Schlußstein einer profanen Heilsgeschichte - die zudem, ganz im Geist des Renaissancis-

15 Erwin Panofsky/Fritz Saxl: Classical Mythology in Medieval Art, in: Metropolitan Museum Studies IV, 1933, $228 \mathrm{ff}$.

16 Dieter Blume: Regenten des Himmels. Astrologische Bilder in Mittelalter und Renaissance, Berlin 2000, $2-3$.

17 Warburg 1998 (wie Anm. 1), 467. 
mus, als Antidot zu einer als veränderungsbedürftig erachteten Gegenwart verstanden wird.

Ferrara freilich war bis anhin im kunsthistorischen Heilsplan durchaus nicht vorgekommen. Giorgio Vasari streift die dortige Malerei nur kursorisch, über die Fresken des Palazzo Schifanoia schweigt er sich ganz aus. Seit diese im Jahre 1710 übertüncht worden sind, bricht die Überlieferung fast gänzlich ab; erst ihre Freilegung im 19. Jahrhundert hat sie wieder ins Blickfeld geraten lassen. Jakob Burckhardt gehört zu den ersten, die das Freskenprogramm eingehender würdigen; wobei er mit der Zuschreibung eines Teils der Fresken an Piero della Francesca sich mehr auf die Kunstliteratur (namentlich Luigi Lanzi) denn auf den eigenen Augenschein verlassen zu haben scheint. ${ }^{18}$

Erst die im Jahre 1933-1934 ausgerichtete Ausstellung zur Ferrareser Malerei des 14 . bis 16. Jahrhunderts hat eine größere Öffentlichkeit dann wirklich mit den Meistern des Palazzo Schifanoia und deren Umfeld bekannt gemacht. Sie nahm Roberto Longhi zum Anlaß, eine erste, eigene Geschichte dieser Malschule zu verfassen. So wie später, unmittelbar nach dem Krieg, gelegentlich der venezianischen Ausstellung Mostra di cinque secoli di pittura veneta der Viattico della pittura veneziana entstehen sollte - als eine Art Antikatalog ${ }^{19}$ schreibt Longhi auch hier in strikter, an der eigenen Kennerschaft orientierten Chronologie an den Exponaten entlang, bestätigt oder verwirft Zuschreibungen, adjektiviert (mit seinen „equivalenze verbali“ ${ }^{{ }^{20} \text { ) }}$ sein Kunsterlebnis, zieht nicht ausgestellte Werke heran - darunter die Fresken des Palazzo Schifanoia -, um das Bild zu komplettieren, und schafft so, in einem Zug und atemloser Prosa, die Geschichte eines unvergleichlichen visuellen Laboratoriums: die Officina Ferrarese.

Auf den primordialen literarischen Rang Longhis, den Ezio Raimondi, neben Carlo Emilio Gadda zu den Hauptvertretern des „Barocco Moderno“ zählt, kann hier nicht eingegangen werden. ${ }^{21}$ Longhi mag viele Anlässe für dieses

18 Jakob Burckhardt: Der Cicerone. Eine Anleitung zum Genuß der Kunstwerke Italiens, in: ders., Kritische Gesamtausgabe, hrsg. v. Bernd Roeck u.a., München/Basel 2001, Bd. 1, 177; Bd. II, 79, 81 .

19 Roberto Longhi: Viatico per cinque secoli di pittura veneziana, Florenz 1946 (deutsche Ausgabe: Venezianische Malerei, Berlin 1995). Vgl. dazu Andreas Beyer: Renaissance Studies. Between Academic and Exhibition Practice, in: Charles W. Haxthausen (Hrsg.), The Two Art Histories. The Museum and the University (Clark Studies in the Visual Arts), New Haven/London 2002, 25-31.

20 Vgl. dazu Giovanni Previstali (Hrsg.): L'arte di scrivere sull'arte. Roberto Longhi nella cultura del nostro tempo, Rom 1982 sowie Andreas Beyer: Roberto Longhi, in: Heinrich Dilly (Hsrg.), Altmeister moderner Kunstgeschichte, Berlin 1990, 251-265.

21 Vgl. Previtali 1982 (wie Anm. 20); Corrado Bologna: Officina Ferrarese di Roberto Longhi, in: Alberto Asor Rosa (Hrsg.), Letteratura Italiana. Le opere. Bd. IV, Il Novecento, II: La ricerca letteraria, Turin 1996, 3-58. 
Buch gehabt haben. Nicht zuletzt geht es hier um seinen Kampf mit und gegen Bernard Berenson, um die Zuschreibungshoheit auch im Bereich der mittelitalienischen Malerei: - etwa zwanzig Arbeiten des Ercole de' Roberti (und damit die Hälfte des seinerzeit konstituierten (Euvres) löst Longhi aus Berensons Katalog. ${ }^{22}$

Longhi wird es in diesem Buch, das ein ,Salon' französischer Herkunft aber genuin longhianischer Prägung ist, zunächst und vor allem wohl darum gegangen sein, dem historischen Bewußtsein eine von der Forschung marginalisierte Malschule zu restituieren, sie ins eigene Recht zu setzen und zugleich einzuschreiben in die Teleologie des vasarianischen Kunstschemas - sowie in die eigene bildkritische Kompetenz..$^{23}$ Das Trecento sieht Longhi im Ferraresischen noch ganz durchdrungen von Residuen der höfischen und internationalen Gotik, vor allem aber unter dem dominierenden Einfluß der Malerei von Bologna, Modena oder Padua - „[...] ma nessuna pittura ferrarese propriamente detta. “2+ Erst mit dem Quattrocento, namentlich mit den Meistern Cosmè Tura, Francesco del Cossa und Ercole de' Roberti, erkennt er auch Ferrara zu jener Höhe und Blüte der Kunst gelangen, welche die Stadt konkurrenzfähig gemacht habe mit den anderen Zentren der italienischen Renaissance. Longhi entwirft ein Panorama der Ferrareser Malerei, das sich als Amalgam aus toskanisch-florentinischer, zu Teilen auch venetischer Tendenzen präsentiert und damit als unverbrüchlicher - wenn auch in seiner Eigenart exzentrischer - Teil der apenninischen Malkultur. Unverzichtbar zu deren Ausbildung ist ihm Donatello im nahen Padua, der Einfluß Masaccios weit über Florenz hinaus, die Kenntnis Piero della Francescas, nicht zuletzt die Gestalt Jacopo Bellinis. „Che cosa sortisse da questo incontro di spiriti antichi e di problemi moderni nel temperamento feudale, ghibellino, di Cosmè Tura, cortigiano di Borso d'Este, è a tutti noto. Egli stabilisce nell'arte dell'Italia del Nord la fisionomia specifica dell'arte ferrarese. È il primo genio del luogo."

22 Longhi 1975 (wie Anm. 2), 59. Vgl. zu Longhi und Berenson auch Cesare Garbali/Cristna Montagnani: Bernard Berenson - Roberto Longhi. Lettere e scartafacci, Mailand 1993.

23 Ein frühes, eindrucksvolles Zeugnis der Wirkung der Longhianischen Prosa und seines Anspruches auf Wortführerschaft stellt die Besprechung der Officina Ferrarese von Ellis K. Waterhouse im Burlington Magazine 3, 1936, 150-151 dar: „Aternate anger and delight will possess the reader of this book; delight on almost every page at the acuteness of Professor Longhi's mind and the wideranging flights of his sensibility, anger more rarely, but in pungent gusts, at his incapacity or unwillingness to control these intemperate virtues $[\ldots]$ It will be clear from this summary that the Directors of Art Institutes will have to put this book under lock and key: it is too full of inflammable material for the unfledged mind and too irresponsible in its bright ideas. But they will have to get it." Vgl. auch Longhis lakonische Reaktion in Longhi 1975 (wie Anm. 2), 151.

24 Longhi 1975 (wie Anm. 2), 13.

25 Ebd., 31. 
Und so wie er Tura als einen „Pollaiolo aculeato ${ }^{26}$ umschreibt, als stacheliges, dorniges Pendant zu seinem Florentiner Zeitgenossen, stellt er diesen und die gesamte Ferrareser Renaissance in wirksame Abhängigkeit von der toskanischen Kunstmetropole. In nuce ist dieses (kunst-) geschichtsphilosphische Entwicklungsmodell in folgender Passage enthalten: „Tutto il resto dell'opera è al sommo consistente, ferocemente eguale. Organismo, consigliano i fiorentini nell'architettura e il Tura crea il superorganismo della sua architettura che dà nell'assiro e nel salomonico. Il simile avviene degli altri regni del creato sotto il piglio del fiero maestro. Un'ascendenza medievale lo convince preventivamente che non sia pittura se prima non si concreti in un materiale raro ed eletto (il misticismo medievale delle pietre e delle gemme); si immagini che cosa ne consegue al contatto dei principî organici venuti di Toscana. Sì, potenza di moto negli uomini negli alberi nelle rocce, ma che, nel materiale immaginato dei minerali più incorruttibili, non può che torcersi e serrarsi, quasi in turbini impietrati. Una natura stalagmitica; un'umanità di smalto e di avorio con giunture di cristallo. Anche il ricordo di Piero fa l'ufficcio suo e subito si tramuta. Sui celi di lapisazzurro incrinano figure smeraldine o arrubinate agli orli; i tramonti sono di croco ossificato. Come un astrologo il Tura escogita forme, predilige oggetti che sian simboli pregnanti del suo sogno stilistico. Conchiglie, buccine, perle, tritoni, gracole, draghi, grotte, origlieri sono alcuni di questi suoi stemmi. Nulla mi ha meglio appreso sopra lo spirito zodiacale del Tura che il veder, dalla nube sopra il Roverella in ginocchio, coagulata nel celo di tramontana già denso come un bicchiere di ginepro, spuntare l'unicorno estense. "27 „Aszendenzen“; „Astrologe“: Longhi, hier selbst Seher und Deuter der Malereien des Tura, brauchte nicht Warburg gelesen zu haben, um diesen künstlerischen Kosmos in der Nomenklatur der Astrologie zu vermessen. In Turas souveränen $\mathrm{Zu}$ - und Rückgriffen erhebt er den Maler zum Schöpfer einer Kunstwelt, die als veritable ,Ausgründung ' der Florentinischen Schule erscheint, als deren Fortsetzung in einem schöpferischen, bald sich verselbständigenden, erst durch seinen Ursprung aber faßbaren Delirium.

Warburg und Longhi konvergieren in dem Bemühen, die rätselhafte Faszination Ferraras in dem Maße zu beherrschen, und damit zu entkräften, indem sie diese in die Traditionslinien der Astrologie und Mythographie einerseits, in die Kräftelinien der italienischen Malerei andererseits einschreiben.

Was das astrologische Programm des Palazzo Schifanoia betrifft, wird, so Dieter Blume, leicht vergessen, daß, so zutreffend diese Sicht in mancher Hinsicht sei, sie doch einseitig bleibe: „[...] jene Astrologen am Hof des Borso d'Este gar nicht in der Lage waren, in dem mit einem Strick gegürteten Mann 
den verkleideten Perseus zu erkennen. “28 Und aus dem Blick gerate, „(..) daß es gerade nicht das Interesse an der Antike war, das sie zur Darstellung dieser Figur bewegte, sondern die Bewunderung der überlegenen arabischen Astrologie, die gegen die mythographische Überlieferung des Westens ausgespielt wurde." $" 29$

Und auch Longhis stil- und formanalytische Einvernahme der Ferrareser Fresken kommt der Eigenart, der spezifischen Konsistenz dieser Malerei kaum entgegen. Zudem sind seine Ergebnisse im Detail, wie im Falle Warburgs, nicht unwidersprochen geblieben. Nicht überzeugt hat seine Rekonstruktion des Roverella-Polyptychons; die von Longhi vaterlos gemachten Werke Turas oder de' Robertis sind zum Teil wieder reattribuiert worden. ${ }^{30}$ Allenfalls hat sich Longhis Erfindung des „Vicino da Ferrara“ (ein Notname, der im „Vicino“ seine Nähe zu den Ferrareser Meistern artikuliert) durchgesetzt. Und doch hat er so folgenreich gewirkt, daß die bereits im 18. Jahrhundert geäußerte Vermutung, etwa Cosmè Tura könne sich an nördlicher Malerei orientiert haben ${ }^{31}$, nie wieder Ansatzpunkt einer Forschung gewesen ist, die nach anderen Verbindungssträngen gesucht hätte, nach weiteren, irritierenderen, komplexeren Bedingungen. Das Projekt einer wider den Strich gebürsteten Geschichte der italienischen Kunst, die sie als Ergebnis stetiger äußerer Beeinflussung betrachtete, stellt ja noch immer das große Desiderat der Italienforschung dar.

Longhis Officina darf verstanden werden als das Korrelat zu Warburg; der ja vom „enthusiastische[n] Staunen vor dem unbegreiflichen Ereignis künstlerischer Genialität" spricht und die Form zum kongenialen Träger der befreiten Tradition erklärt: „Der neue große Stil, den uns das künstlerische Genie Italiens beschert hat, wurzelte in dem sozialen Willen zur Entschälung griechischer Humanität aus mittelalterlicher, orientalisch-lateinischer ,Praktik'. "3:2 Erst mit der Renaissance freilich bricht - in der Sicht Warburgs nicht anders, als in jener Longhis - die Epoche an, in der diese Schlacht um Aufklärung erfolgreich geschlagen wurde.

Stellten wir uns eine Begegnung Warburgs und Longhis, die in ihren Schriften niemals Bezug aufeinander genommen haben, in Ferrara vor, dann ließe sich zuversichtlich erwarten, daß beide in einen enthusiastischen Dialog einträten: ein Gespräch, das, bei aller unterschiedlichen schulischen Prägung, beide sich hätten auf eine Kunstgeschichte der Herleitung und Zuschreibung einigen lassen, auf das gemeinsame Konzept einer humanistischen Eschatologie, das

28 Blume 2000 (wie Anm. 16), 2.

29 Ebd., 3.

30 Vgl. Waterhouse 1936 (wie Anm. 23), 151.

31 „Al suo piegare si assomigliò molto AJberto Durero“, so C. Barotti in seinem Werk Pitture e sculture della città di Ferrana, 1770.

32 Warburg 1998 (wie Anm. 1), 479. 
den hermetischen Kosmos Ferraras aufgesprengte und der Geistes- und Formgeschichte der Renaissance einschriebe. Und so wie Albert Camus sich den Sisyphos dachte, so müßten wir uns beide dabei als glückliche Menschen vorstellen - Vita brevis, ars longhi.

Bildnacbweis: Geheimes Wissen. Verlorene Techniken der Aiten Meister wieder entdeckt von David Hockney, London/München 2001, 18. 